\section{Indikation einer Laboruntersuchung}

\author{
A. M. Gressner ${ }^{1}$ und O. A. Gressner ${ }^{2}$ \\ ${ }^{1}$ Labor Dr. Wisplinghoff Berlin, Berlin, Deutschland \\ ${ }^{2}$ Labor Dr. Wisplinghoff Köln, Köln, Deutschland
}

Synonym(e) Labormedizinische Indikationsstellungen

\section{Englischer Begriff test purpose}

Definition Auswahl und Veranlassung der Bestimmung einer klinisch-chemischen bzw. labormedizinischen Kenngröße zum Zwecke von Diagnose, Verlaufskontrolle, Prognosebeurteilung und Prädispositionsdiagnostik.

Beschreibung Bei der Auswahl klinisch-chemischer Kenngrößen ( $\triangleright$ Kenngröße, klinisch-chemische) zum Zwecke der Diagnostik sind Kenntnisse zu > Spezifität, diagnostische; - Sensitivität, diagnostische; > Vorhersagewert, negativer und $>$ Vorhersagewert, positiver und zu Merkmalen der Präanalytik ( $\triangleright$ Einflussgrößen, \ Störgrößen) notwendig, um eine auf die klinische Fragestellung bezogene Validitätsbeurteilung zu ermöglichen. Die Auswahl durch den Arzt basiert auf dessen klinischen, pathobiochemischen und labormedizinischen Wissensstand und Erfahrungen, dem klinischen Zustand des Patienten, der klinischen Fragestellung für Diagnostik, Verlaufskontrolle, Prognosebeurteilung und Prädispositionsdiagnostik (z. B. Risikofaktoren) und auf den technischen, personellen und ökonomischen Bedingungen, die mit der Erstellung der klinisch-chemischen Kenngröße verbunden sind.

\section{Literatur}

Casscells W, Schoenberger A, Graboys TB (1978) Interpretation by physicians of clinical laboratory results. N Engl J Med 299:999-1001

Hindmarsh JT, Lyon AW (1996) Strategies to promote rational clinical chemistry test utilization. Clin Biochem 29:291-299 